\title{
Alignment-dependent ionization of hydrogen molecules in intense laser fields
}

\author{
Ying-Jun Jin,,$^{1, *}$ Xiao-Min Tong, ${ }^{1,2, \dagger}$ and Nobuyuki Toshima ${ }^{1}$ \\ ${ }^{1}$ Institute of Materials Science, Graduate School of Pure and Applied Sciences, University of Tsukuba,1-1-1 Tennodai, \\ Tsukuba, Ibaraki 305-8573, Japan \\ ${ }^{2}$ Center for Computational Sciences, University of Tsukuba,1-1-1 Tennodai, Tsukuba, Ibaraki 305-8577, Japan
}

(Received 12 April 2011; published 14 June 2011)

\begin{abstract}
Recent experiments showed that the alignment-dependent ionization probabilities of hydrogen molecules vary as a function of laser intensity, and the anisotropy deviates from the prediction of the molecular tunneling ionization model (MO-ADK). To investigate the physical origins of the deviation, we systematically studied the anisotropy of hydrogen molecules in intense laser fields for three wavelengths, $400 \mathrm{~nm}$ (multiphoton ionization), $1850 \mathrm{~nm}$ (tunneling ionization), and $800 \mathrm{~nm}$ (in between), by solving the time-dependent Schrödinger equation with a model potential. The calculated ratio of the ionization probabilities for laser fields parallel and perpendicular to the molecular axis are in reasonable agreement with experiment. Furthermore, by analyzing the molecular wave function and the model potential, we found that the discrepancies between experiment and the MO-ADK prediction originated from the inaccurate coefficients used in the model and that the assumption of isotropy of the effective potential in the tunneling region is invalid.
\end{abstract}

DOI: 10.1103/PhysRevA.83.063409

PACS number(s): $33.80 . \mathrm{Rv}, 42.50 . \mathrm{Hz}, 34.50 . \mathrm{Gb}$

\section{INTRODUCTION}

Multiphoton ionization of atoms and molecules in intense laser fields is an important research subject and has been extensively studied both experimentally $[1-3]$ and theoretically [4-6]. When the Keldysh parameter [7] is smaller then unity, the ionization probability can be estimated by the atomic tunneling ionization model [8] and the molecular tunneling ionization model (MO-ADK) [9]. Based on the MO-ADK, the molecular tunneling ionization probability depends on the alignment of the molecular axis with respect to the laser field, and the ionization probabilities mimic the valence electron density distributions at the tunneling position. The electron density distributions were observed experimentally for several molecules [10-12]. These all stimulated more experimental [13,14] and theoretical [15-17] studies on the molecular ionization in intense laser fields. In these experiments, people measured the Coulomb explosive products induced by the collision of the rescattering electron [18] with the parent molecular ions [19-21] instead of directly measuring the ejected electron. Most of the observations can be qualitatively explained by the MO-ADK theory.

Recently Staudte et al. [22] measured the angular tunneling ionization probability of $\mathrm{H}_{2}$ molecules using a circularly polarized $800 \mathrm{~nm}$ intense laser and found that the ratio $P_{\|} / P_{\perp}$ varies as a function of laser intensity. The measured ratio is generally larger than that predicted by the MO-ADK theory, which does not depend on the laser intensity nor on the wavelength. Here, $P_{\|}\left(P_{\perp}\right)$ stands for the ionization probability when the laser field is parallel (perpendicular) to the molecular axis. To eliminate the multiphoton contributions, Magrakvelidze et al. [23] measured the ratio $P_{\|} / P_{\perp}$ using a 1850-nm-wavelength laser and found that the ratio is in agreement with the prediction of the MO-ADK theory.

\footnotetext{
*kin@ vivaldi.bk.tsukuba.ac.jp

†tong.xiaomin.ga@u.tsukuba.ac.jp
}

Chu [24] has studied the strong-field ionization rates of $\mathrm{H}_{2}$ molecules using time-dependent density functional theory (TDDFT). She found that, in the tunneling ionization limit, the results of TDDFT are in agreement with the MO-ADK predictions but the parameters used for $\mathrm{H}_{2}$ should be revised. Note that the ratio of TDDFT depends on the laser intensity. Given all these facts, we may naturally ask what is the validity region of the MO-ADK, how large is the contribution of the multiphoton process, and which assumption used in the MO-ADK theory is inappropriate?

To address these questions, we investigated the alignmentdependent ionization probability of $\mathrm{H}_{2}$ in intense laser pulses for three wavelengths solving the time-dependent Schrödinger equation in prolate spheroidal coordinates with a model potential. The wavelengths used in the simulations are $400 \mathrm{~nm}$, which stands for multiphoton ionization, $1850 \mathrm{~nm}$, which stands for the tunneling ionization region, and $800 \mathrm{~nm}$, which stands for the coexistence of both processes. By analyzing the electron density distribution and the isosurface of the model potential, we showed that the coefficients used in the MO-ADK model are sensitive to the position where we define the asymptotical region and the coefficients should be revised as suggested by Chu [24]. Furthermore, the decrease of the ratio $P_{\|} / P_{\perp}$ as a function of laser intensity can originate partly from the anisotropy of the model potential in the tunneling region. We present the numerical method in Sec. II and the results and discussion in Sec. III.

\section{THEORETICAL METHOD}

To study the alignment-dependent ionization of diatomic molecules, we extended our previous two-dimensional timedependent generalized pseudospectral method [25] to a threedimensional one. We studied the system in prolate spheroidal coordinates, which can properly describe a two-center problem. The time evolution of the single-electron wave function is governed by the following time-dependent Schrödinger 
equation as (atomic units $\hbar=m=e=1$ are used through the paper unless stated otherwise)

$$
i \frac{\partial}{\partial t} \Psi(t)=\left[H_{0}+V_{\mathrm{ext}}(t)\right] \Psi(t) .
$$

The electron laser interaction $V_{\text {ext }}(t)$ is written as

$$
V_{\mathrm{ext}}(t)=F(t)\left[a \xi \eta \cos \theta+a \sqrt{\left(\xi^{2}-1\right)\left(1-\eta^{2}\right)} \cos \varphi \sin \theta\right],
$$

where $a=R / 2$ with $R$ being the distance between the two nuclei, and $\theta$ being the angle between the laser polarization direction and the molecular axis. $F(t)$, the linear polarized time-dependent laser field strength, is written as

$$
F(t)=E_{0} e^{-2 \ln 2 t^{2} / \tau^{2}} \cos (\omega t) .
$$

where $E_{0}$ is the peak field strength, $\tau$ is the full width at half maximum of the pulse duration, and $\omega$ is the center frequency of the intense laser. $\xi, \eta$, and $\varphi$ are prolate spheroidal coordinates, which are related to Cartesian coordinates $x, y$, and $z$ as

$$
\begin{gathered}
x=a \sqrt{\left(\xi^{2}-1\right)\left(1-\eta^{2}\right)} \cos \varphi, \\
y=a \sqrt{\left(\xi^{2}-1\right)\left(1-\eta^{2}\right)} \sin \varphi, \\
z=a \xi \eta .
\end{gathered}
$$

$H_{0}$, the laser-field free model Hamiltonian of a diatomic molecule, is given as

$$
H_{0}=-\frac{1}{2} \nabla^{2}+V(\xi, \eta)+\frac{1}{2 a},
$$

where $V(\xi, \eta)$ is a model potential. Since $H_{0}$ has rotational symmetry with respect to the molecular axis, the timeindependent wave function can be written as

$$
\psi(\xi, \eta, \varphi)=\Phi^{m}(\xi, \eta) e^{i m \varphi} .
$$

We discretized $\xi, \eta$ using the generalized pseudospectral method [26] and propagated the time-dependent wave function by solving the time-integral equation

$$
\begin{aligned}
\Psi(t)= & -i \int_{t_{0}}^{t} e^{-i \int_{\tau}^{t}\left[H_{0}+V_{\mathrm{ext}}\left(t^{\prime}\right)\right] d t^{\prime}} V_{\mathrm{ext}}(\tau) e^{-i H_{0} \tau} \Phi_{0} d \tau \\
& +e^{-i H_{0} t} \Phi_{0}
\end{aligned}
$$

by the second-order split-operator method in the energy representation [27]. Here $\Phi_{0}$ is the ground-state wave function. The above time-integral equation is equivalent to Eq. (1) mathematically, but can be solved with higher numerical accuracy because we factored out the last term (background), which has no contribution to the laser-induced processes. The time-integral equation has been successfully applied to study collision problems [28,29] and dynamical problems of atom-intense-laser interactions [30,31]. The former can also be treated as time-independent problems, whereas the latter can only be treated by the time-dependent method. We added an optical absorber to remove the outgoing electron when it reaches the outer region. When the laser pulse is over, the sum of the electron in the continuum and the electron removed by the optical absorber gives the ionization probability for a given alignment.
For $\mathrm{H}_{2}$, we chose the model potential

$$
V(\xi, \eta)=V_{\mathrm{eff}}\left(r_{1}\right)+V_{\mathrm{eff}}\left(r_{2}\right),
$$

where $r_{1}\left(r_{2}\right)$ is the distance between active electron and nucleus one (two) and

$$
V_{\mathrm{eff}}(r)=-\frac{\alpha+\beta e^{-\sigma r}}{r},
$$

with $\alpha=0.5, \beta=0.5$, and $\sigma=1.78$. Here we fixed the internuclear distance at $R=1.4$ a.u. The criteria for choosing the model potential were that (1) the ionization potential obtained from the model potential is close to the measured one $(15.43 \mathrm{eV}) ;(2)$ the electron feels the correct asymptotic Coulomb interaction when it is far from the molecular core; and (3) the electron feels the pure Coulomb interaction of a proton when it is close to one of the nuclei. The above model potential satisfies all the requirements. The numerical convergence has been checked by varying the number of grid points and box size.

\section{RESULTS AND DISCUSSION}

Based on the above theoretical method, we investigated the ratio $P_{\|} / P_{\perp}$ for $\mathrm{H}_{2}$ at three laser wavelengths for an intensity range from 1 to $4 \mathrm{I}_{0}$ with $I_{0}=1.0 \times 10^{14} \mathrm{~W} / \mathrm{cm}^{2}$ and for different pulse durations. For a longer pulse duration, the molecules can be completely ionized at such an intensity. As the laser intensity becomes higher, the ionization probabilities as well as the ratio approach unity. Note that the ratio of ionization rates predicted by the MO-ADK theory [9] is 1.17 and the value does not depend on the laser intensity nor on the wavelength.

The ionization probability $P(\theta)$ can be expressed as

$$
P(\theta)=1-e^{-W(\theta) \tau},
$$

where $\theta$ is the alignment angle, $W(\theta)$ is the ionization rate, and $\tau$ is the pulse duration of the laser field. Figure 1 (upper panel) shows the ratio of ionization rates $\left(W_{\|} / W_{\perp}\right)$ obtained from the ionization probabilities for a $1850 \mathrm{~nm}$ laser field. The ratio weakly depends on the pulse duration and decreases as the laser intensity increases. The ratio is higher than that predicted by the MO-ADK theory. The lower panel of the figure shows the calculated ratio $P_{\|} / P_{\perp}$ in comparison with the experimental value [23]. The pulse duration of the experiment is $50 \mathrm{fs}$ so we estimated the $50 \mathrm{fs}$ ratio using the ionization rates obtained from the 20 fs results. As the laser intensity increases, the ratio decreases from 1.25 to 1.14 for a 10 fs pulse. For lower intensities, the ratio does not depend much on the pulse duration. For higher intensities, the ratio decreases as the laser pulse duration increases. This can be explained by the fact that the ionization probability is very small and almost proportional to the pulse duration for lower intensities. For higher intensities and longer pulses, the molecules are almost completely ionized for any alignment and the ratio $P_{\|} / P_{\perp}$ becomes closer to unity. Our results are in reasonable agreement with experiment when we use the same pulse duration (50 fs) as the experimental one. The present results are also higher than the MO-ADK value in the lower-intensity region and approach it in the higherintensity region. For $1850 \mathrm{~nm}$, the Keldysh parameter is about 0.5 at $\mathrm{I}_{0}$, which indicates that tunneling ionization is dominant. 

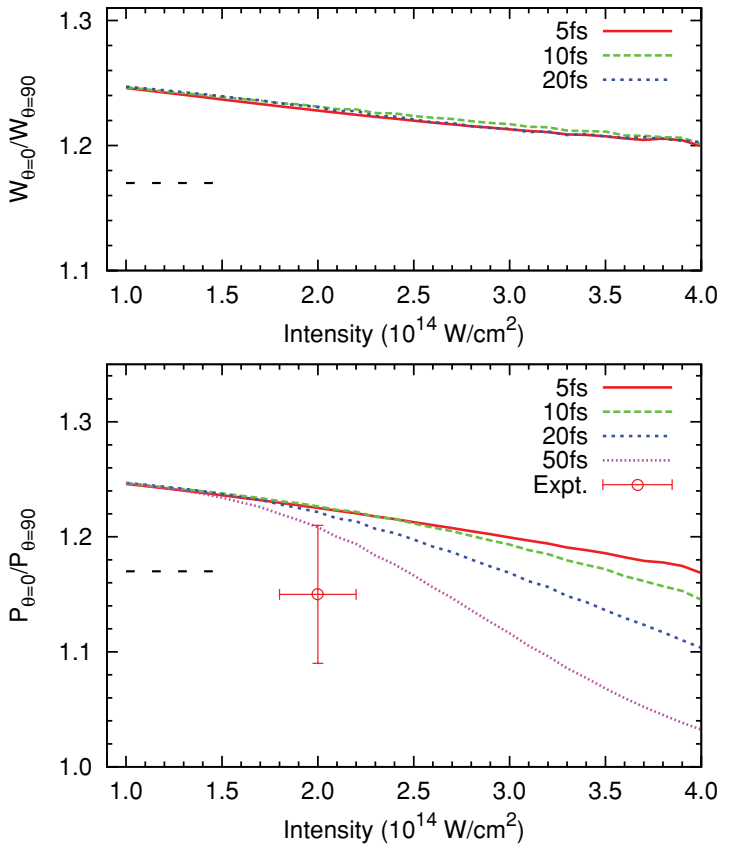

FIG. 1. (Color online) Ratio of ionization rates $\left(W_{\|} / W_{\perp}\right.$, upper panel) and of ionization probabilities $\left(P_{\|} / P_{\perp}\right.$, lower panel) for $\mathrm{H}_{2}$ in $1850 \mathrm{~nm}$ laser fields for different pulse durations. The MO-ADK value is indicated by the horizontal dashed line and the internuclear distance is fixed at the equilibrium distance $R=1.4$.

For an $800 \mathrm{~nm}$ laser, the Keldysh parameter is 1.15 at $\mathrm{I}_{0}$ and tunneling ionization should be important for laser intensities higher than $\mathrm{I}_{0}$. Figure 2 shows the calculated ratio $P_{\|} / P_{\perp}$ and the experimental values [22]. Note that the laser intensity of the experiment is divided by 2 since a circularly polarized laser was used in the experiment. The pulse duration of the experiment is $40 \mathrm{fs}$ so we estimated the 40 fs ratio by using the ionization rates obtained from the $20 \mathrm{fs}$ results. At lower intensities, the ratio is slightly higher than that found at $1850 \mathrm{~nm}$. The general trend of the pulseduration dependence is similar; namely, the ratio becomes smaller for longer pulse durations at higher intensities. Our results for the long pulse (40 fs) are in good agreement with the experimental results.

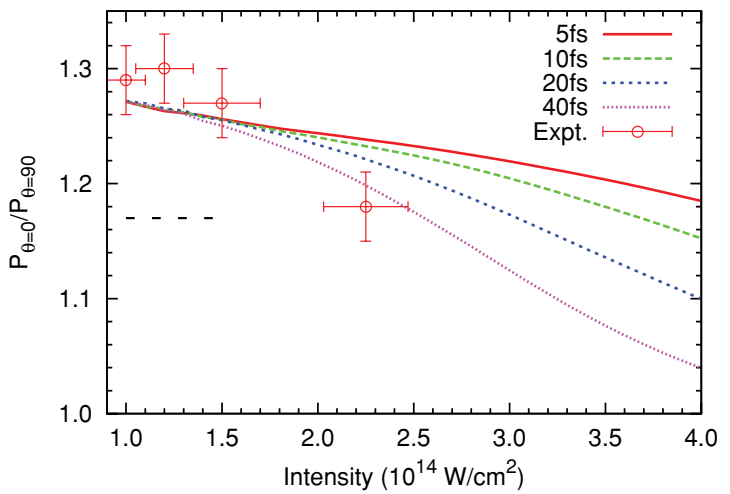

FIG. 2. (Color online) Ratio $P_{\|} / P_{\perp}$ for $\mathrm{H}_{2}$ molecules in $800 \mathrm{~nm}$ laser fields for different pulse durations. The MO-ADK value is indicated by the horizontal dashed line and the internuclear distance is fixed at the equilibrium distance $R=1.4$.

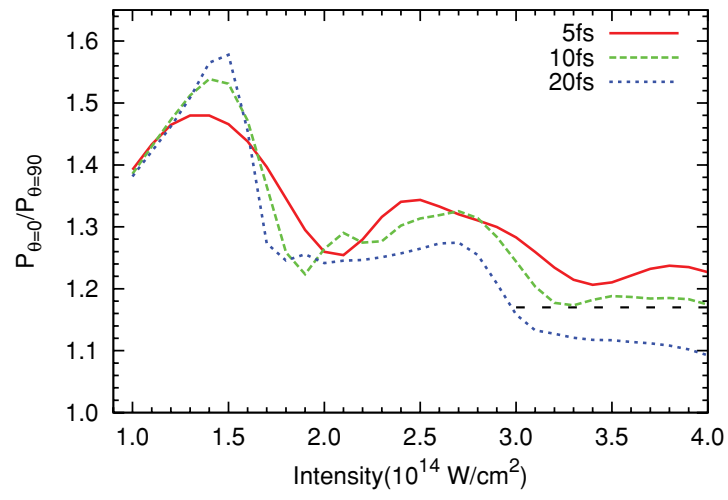

FIG. 3. (Color online) Ratio $P_{\|} / P_{\perp}$ for $\mathrm{H}_{2}$ molecules in $400 \mathrm{~nm}$ laser fields for different pulse durations. The MO-ADK value is indicated by the horizontal dashed line. The internuclear distance is fixed at the equilibrium distance $R=1.4$.

Now we look at the $400 \mathrm{~nm}$ case, for which the Keldysh parameter is much larger than unity. This means multiphoton ionization is dominant. Figure 3 shows the calculated ratio $P_{\|} / P_{\perp}$. Similar to the longer-wavelength cases, the ratio is always larger than unity. The ionization is favorable to the case when the laser field is parallel to the molecular axis. Unlike the longer-wavelength cases, the ratio oscillates as a function of laser intensity. For multiphoton ionization, the ionization probability is sensitive to the electron energy structure and a small ponderomotive shift may significantly affect the probability. For higher intensities, tunneling ionization becomes important, the oscillation amplitude reduces, and the ratio approaches that predicted by the MO-ADK theory.

In the above analysis, we saw that the MO-ADK theory works only for higher laser intensities [the horizontal dashed lines in Figs. (1-3)]. To investigate the validity region of the MO-ADK theory and the accuracy of the coefficients used in it, we plotted the electron densities in the directions along and perpendicular to the molecular axis in Fig. 4. Based on the concept of the MO-ADK theory, the ionization rate along a given direction is proportional to the electron density in that direction at the tunneling position. In the MO-ADK theory, to simplify the calculation of the electron density at the tunneling

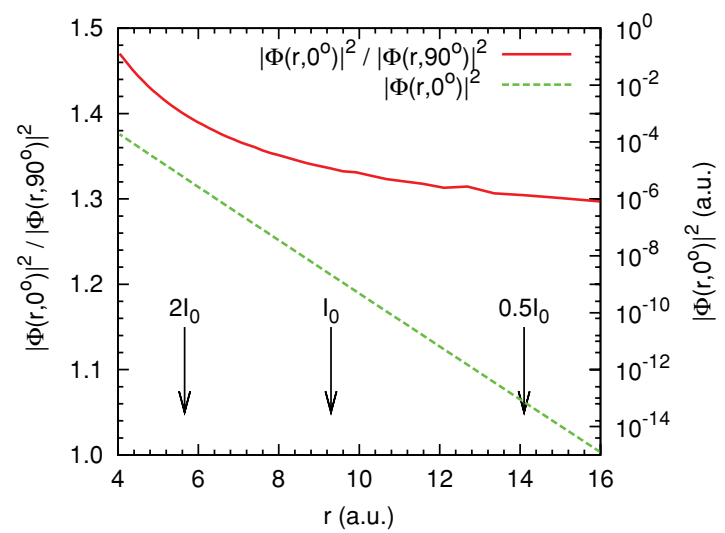

FIG. 4. (Color online) Ratio of electron densities between the parallel and perpendicular directions (solid line) and the electron density along the molecular axis (dashed line). The tunneling positions for given laser intensities are indicated by vertical arrows. 


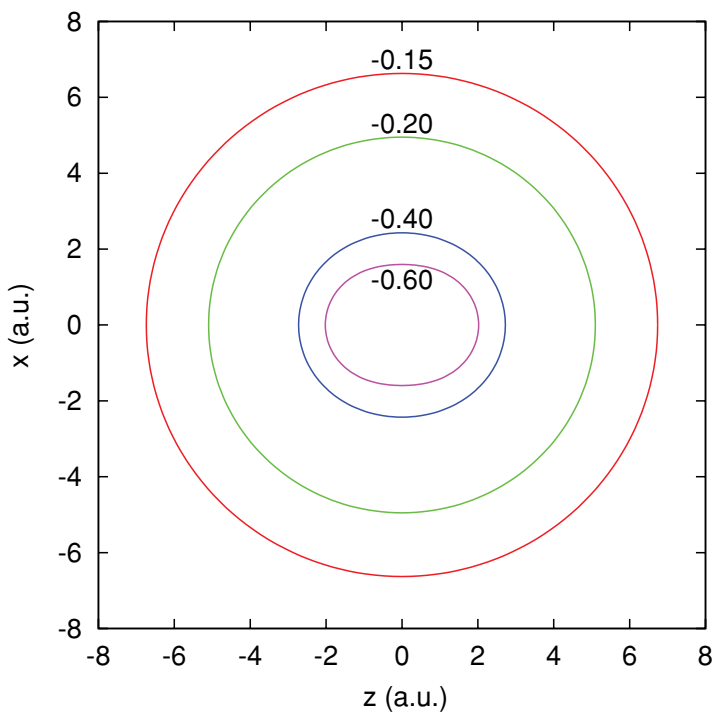

FIG. 5. (Color online) Isosurface of the model potential in the $x-z$ plane, with $z$ along the molecular axis. The numbers indicate the isopotential values in atomic units.

position, we assume that the electron-core interaction is a pure Coulomb interaction and the electron density at the tunneling position is expressed analytically with coefficients which do not depend on the tunneling position. The coefficients are obtained by fitting the electron density of the ground state in the asymptotic region. Since the electron density drops exponentially (as shown in Fig. 4) in the asymptotic region, it is rather difficult to accurately calculate the wave function. The original MD-ADK parameters for $\mathrm{H}_{2}$ are obtained by the multiple-scattering method [32-34] at a distance about 10 a.u. from the center of molecules. As shown in Fig. 4, the electron density drops by several orders of magnitude and the values may not be very accurate. Recently, Chu [24] suggested revising the parameters by more accurate calculations and the predicted ratio of $P_{\|} / P_{\perp}$ is about 1.4, which is consistent with the ratio of densities at a distance of 10 a.u., as shown in Fig. 4. The ratio of densities increases as the distance from the center of the molecule decreases. This suggests that the ratio $P_{\|} / P_{\perp}$ should increase as the laser intensity increases. The conclusion conflicts with experimental observations and our calculations, which show that the ratio decreases as the laser intensity increases.

In order to solve this puzzling problem, we go back to the assumptions used in the MO-ADK equation. To derive the MO-ADK rate, we assumed that the interaction of the electron with the parent molecular ion is a pure Coulomb interaction with a point effective charge at the center of the molecules. The natural consequences of this assumption for a given field strength are (1) the tunneling ionization happens at the same distance from the point charge and (2) the tunneling cone is unchanged at any direction so that only the electron density in that direction plays a role. Figure 5 shows the isosurface of the model potential used in the present simulations. At a large distance from the center of molecules, the isosurface of the model potential is close to a sphere. As the distance from the center of molecules decreases, the isosurface of the model potential approaches an ellipse with the long axis along the molecular axis. Thus, the curvature of the tunneling point along the long axis is larger than that along the short axis, so the tunneling cone along the long axis is smaller than that along the short axis. This effect enhances the ionization probability when the laser field is perpendicular to the molecular axis. This may explain why the ratio $P_{\|} / P_{\perp}$ decreases as the intensity increases since the tunneling point becomes closer to the molecular center.

To summarize, we have studied the alignment-dependent ionization of $\mathrm{H}_{2}$ molecules in pulsed laser fields by solving the time-dependent Schrödinger equation with a model potential. The calculated ratios of the ionization probabilities for laser fields parallel and perpendicular to the molecular axis are in reasonable agreement with experiments. More importantly, we identified a defect of the MO-ADK theory, which predicts the intensity-independent anisotropy of the ionization probabilities.

\section{ACKNOWLEDGMENTS}

This research was supported by Grand-in-Aid for Scientific Research (C) from the Japan Society for the Promotion of Science.
[1] C. Guo, M. Li, J. P. Nibarger, and G. N. Gibson, Phys. Rev. A 58, R4271 (1998).

[2] M. J. De, Witt E. Wells, and R. R. Jones, Phys. Rev. Lett. 87, 153001 (2001).

[3] E. P. Benis, J. F. Xia, X. M. Tong, M. Faheem, M. Zamkov, B. Shan, P. Richard, and Z. Chang, Phys. Rev. A 70, 025401 (2004).

[4] T. Otobe, K. Yabana, and J. I. Iwata, Phys. Rev. A 69, 053404 (2004).

[5] T. Bartsch and T. Uzer, J. Phys. B 38, S241 (2005).

[6] T. Brabec, M. Cote, P. Boulanger, and L. Ramunno, Phys. Rev. Lett. 95, 073001 (2005).

[7] L. V. Keldysh, Zh. Eksp. Teor. Fiz. 47, 1945 (1965) [Sov. Phys. JETP 20, 1307 (1965)].
[8] M. V. Ammosov, N. B. Delone, and V. P. Krainov, Zh. Eksp. Teor. Fiz. 91, 2008 (1986) [Sov. Phys. JETP 64, 1191 (1986)].

[9] X. M. Tong, Z. X. Zhao, and C. D. Lin, Phys. Rev. A 66, 033402 (2002).

[10] I. V. Litvinyuk, K. F. Lee, P. W. Dooley, D. M. Rayner, D. M. Villeneuve, and P. B. Corkum, Phys. Rev. Lett. 90, 233003 (2003).

[11] A. S. Alnaser, S. Voss, X. M. Tong, C. M. Maharjan, P. Ranitovic, B. Ulrich, T. Osipov, B. Shan, Z. Chang, and C. L. Cocke, Phys. Rev. Lett. 93, 113003 (2004).

[12] A. S. Alnaser, C. M. Maharjan, X. M. Tong, B. Ulrich, P. Ranitovic, B. Shan, Z. Chang, C. D. Lin, C. L. Cocke, and I. V. Litvinyuk, Phys. Rev. A 71, 031403 (2005). 
[13] K. Hosaka, R. Itakura, K. Yokoyama, K. Yamanouchi, and A. Yokoyama, Chem. Phys. Lett. 475, 19 (2009).

[14] Z. Wu, C. Wu, X. Liu, Y. Deng, Q. Gong, D. Song, and H. Su, J. Phys. Chem. A 114, 6751 (2010).

[15] M. Abu-samha and L. B. Madsen, Phys. Rev. A 81, 033416 (2010).

[16] H. A. Leth, L. B. Madsen, and K. Mølmer, Phys. Rev. A 81, 053410 (2010).

[17] S.-F. Zhao, J. Xu, C. Jin, A.-T. Le, and C. D. Lin, J. Phys. B 44, 035601 (2011).

[18] P. B. Corkum, Phys. Rev. Lett. 71, 1994 (1993).

[19] H. Niikura, F. Legare, R. Hasbani, A. D. Bandrauk, M. Y. Ivanov, D. M. Villeneuve, and P. B. Corkum, Nature (London) 417, 917 (2002).

[20] A. S. Alnaser, T. Osipov, E. P. Benis, A. Wech, B. Shan, C. L. Cocke, X. M. Tong, and C. D. Lin, Phys. Rev. Lett. 91, 163002 (2003).

[21] X. M. Tong, Z. X. Zhao, and C. D. Lin, Phys. Rev. Lett. 91, 233203 (2003).

[22] A. Staudte et al., Phys. Rev. Lett. 102, 033004 (2009).
[23] M. Magrakvelidze, F. He, S. De, I. Bocharova, D. Ray, U. Thumm, and I. V. Litvinyuk, Phys. Rev. A 79, 033408 (2009).

[24] X. Chu, Phys. Rev. A 82, 023407 (2010).

[25] Y.-J. Jin, X.-M. Tong, and N. Toshima, Phys. Rev. A 81, 013408 (2010).

[26] D. A. Telnov and Shih I. Chu, Phys. Rev. A 59, 2864 (1999).

[27] X. M. Tong and S. I. Chu, Chem. Phys. 217, 119 (1997).

[28] X. M. Tong, K. Hino, and N. Toshima, Phys. Rev. Lett. 97, 243202 (2006).

[29] X. M. Tong, K. Hino, and N. Toshima, Phys. Rev. Lett. 101, 163201 (2008).

[30] X. M. Tong, K. Hino, and N. Toshima, Phys. Rev. A 74, 031405 (2006).

[31] X. M. Tong, S. Watahiki, K. Hino, and N. Toshima, Phys. Rev. Lett. 99, 093001 (2007).

[32] D. Dill and J. L. Dehmer, J. Chem. Phys. 61, 692 (1974).

[33] X. L. Liang, X. C. Pan, and J. M. Li, Chin. Phys. Lett. 2, 545 (1985).

[34] X. C. Pan, X. L. Liang, and J. M. Li, Acta Phys. Sin. 36, 426 (1987). 